\title{
Impact of clinical pharmacist in an Indian Intensive Care Unit
}

\author{
Mohamed Hisham, Mudalipalayam N. Sivakumar, Ganesh Veerasekar ${ }^{1}$
}

\begin{tabular}{|c|}
\hline 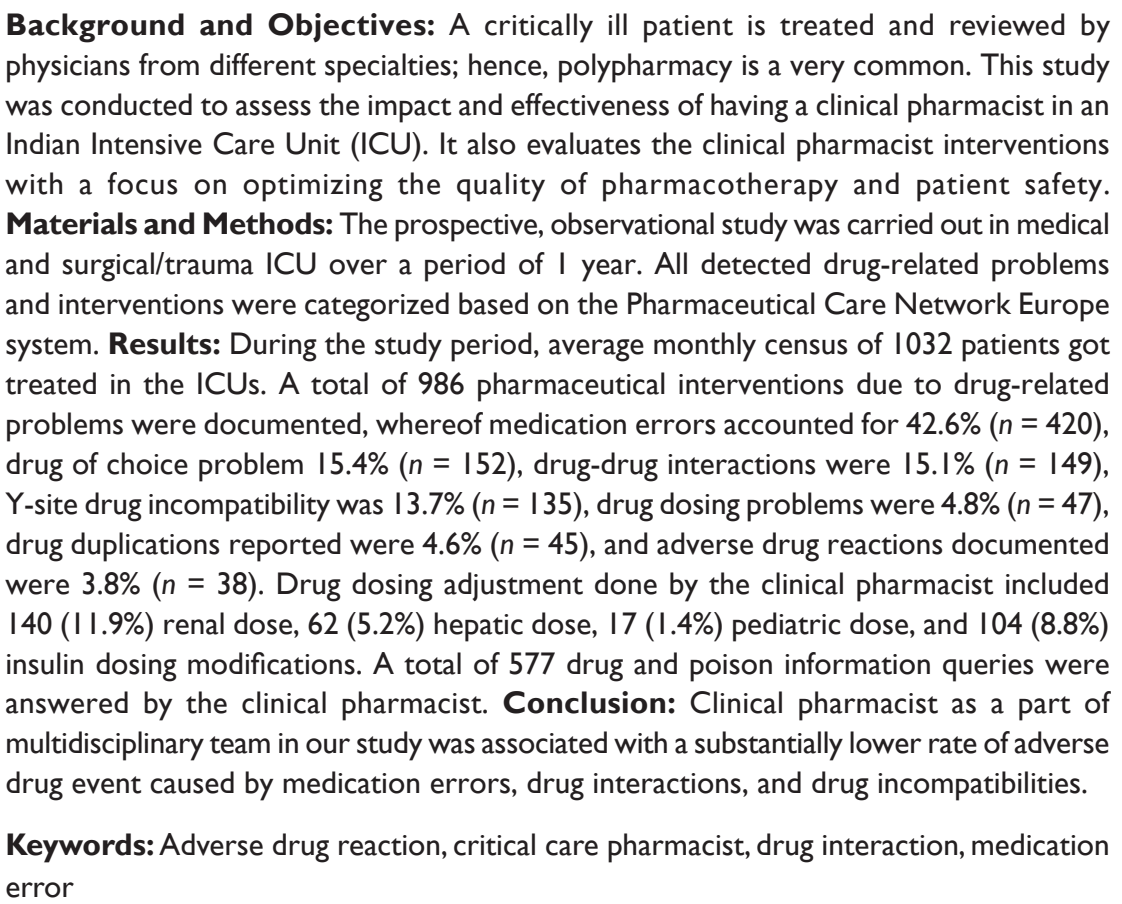 \\
\hline
\end{tabular}

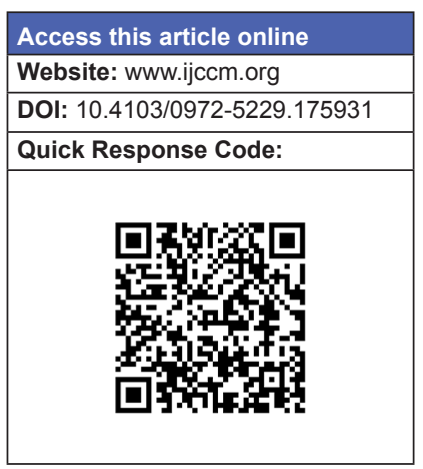

\section{Introduction}

Medication management plays a crucial part in managing a critically ill patient. When it comes to drug therapy, intensivists have plenty of decision making every day including drug selection, dosing, administration, and monitoring strategies to optimize effective pharmacotherapy. Even though the patient receives appropriate drug, a suboptimal dose or overdosing may result in either therapeutic failure

From:

Departments of Critical Care Medicine and ${ }^{1}$ Epidemiology, Kovai Medical

Center and Hospital, Coimbatore, Tamil Nadu, India

\section{Correspondence:}

Dr. Mohamed Hisham, Department of Critical Care Medicine, Kovai Medical Center and Hospital, Avinashi Road, Coimbatore - 641 014, Tamil Nadu, India.

E-mail: hisham_zenith@yahoo.com or drug toxicity. ${ }^{[1]}$ The concept of having a clinical pharmacist in an intensivist-led multidisciplinary team evolved in the early 1980s in USA. ${ }^{[2]}$

Plenty of studies revealed critical care pharmacist involved in a multi-professional healthcare team had constructive impact on the outcome of critically ill patients. In the modern day Intensive Care Unit (ICU),

This is an open access article distributed under the terms of the Creative Commons Attribution-NonCommercial-ShareAlike 3.0 License, which allows others to remix, tweak, and build upon the work non-commercially, as long as the author is credited and the new creations are licensed under the identical terms.

For reprints contact: reprints@medknow.com

How to cite this article: Hisham M, Sivakumar MN, Veerasekar G. Impact of clinical pharmacist in an Indian Intensive Care Unit. Indian J Crit Care Med 2016;20:78-83. 
the skills of a clinical pharmacist are characterized as a responsible caregiver, educator, researcher, and manager. ${ }^{[3]}$ Critical care pharmacist addresses adverse drug events (ADEs) caused due to drug-related problems and medication errors. It improves the appropriateness, quality of prescribing and increases patient safety. Many prospective, controlled trials reveal significantly lower rates of ADEs and lesser days of hospitalization when a clinical pharmacist is integrated into an intensive care healthcare team. ${ }^{[4,5]}$ A study demonstrated reduction in rate of prescribing errors and preventable ADEs by $66 \%$ from 10.4 to 3.5 per 1000 patient days after clinical pharmacist intervention. ${ }^{[6]}$

This study was conducted to assess the impact and effectiveness of having a clinical pharmacist in an ICU. This study aims to evaluate the clinical pharmacist interventions with a focus on optimizing the quality of pharmacotherapy and patient safety. Even though the contribution of critical care pharmacist to improve the quality of patient care is accepted worldwide, many ICUs have not recognized this important reserve. This paper may be used to educate other healthcare professionals and administrators on impact of clinical pharmacist in the care of critically ill patients.

\section{Materials and Methods}

The prospective, observational study was carried out in the medical, surgical/trauma ICU and a high dependency unit at a 750-bedded tertiary care hospital. The ICUs in the study were under the supervision of a clinical pharmacist exclusively for the critical care areas. The study was carried over a period of 1 year from January 1, 2013, to December 31, 2013. The ICUs included in the study together consist of 41 beds. All detected drug-related problems (DRPs) and interventions were categorized based on the Pharmaceutical Care Network Europe (PCNE) system with minor changes according to the applicability in the hospital setting. ${ }^{[7-9]}$

Critical care clinical pharmacy services in our hospital include active participation in physician rounds and review of treatment chart for appropriate indications, drug dosing, dosage form, drug duplication, drug interactions, and drug allergies. Critical care pharmacist plays an important role in identifying, reporting, investigating, and preventing all types of medication errors and adverse drug reactions (ADRs). Other interventions include correction and clarification of physician orders, providing drug and poison information, and recommending an alternative therapy. Clinical pharmacist adjusts dose of drugs in pediatric, geriatric, renal, and hepatic failure patients. Pharmacist also involved in optimizing antibiotic usage based on patient characteristics, site of infection, pharmacokinetics, dose adjustment, and de-escalation. Glycemic control in the ICU is under the surveillance of the clinical pharmacist. Clinical pharmacist also contributes in continuing education activities through teaching programs for doctors and nurses. This increases direct patient care practice abilities, creates awareness among intensive care team, and prevents medication errors.

Data collection was done by the clinical pharmacist on a daily basis and discussed with the ICU chief at the monthly meeting. Data analysis and results were presented as percentage and numerically coded for the ease of descriptive statistics using SPSS software version 20.0 by SPSS Inc., Chicago, IL, USA

\section{Results}

During the study period, average monthly census of 1032 patients got treated in all three ICUs. A total of 986 pharmaceutical interventions due to drug-related problems were documented, of which medication errors accounted for $42.6 \%(n=420)$ followed by $152(15.4 \%)$ drug of choice problem. Manifested or potential drug-drug interactions were 149 (15.1\%) followed by $135(13.7 \%)$ Y-site drug incompatibility, 47 (4.8\%) drug dosing problem, 45 (4.6\%) drug duplication, and $38(3.8 \%)$ ADRs. An average of 0.08 interventions per patient took place during the study period.

All the 986 drug-related problems detected by the clinical pharmacist were categorized based on the PCNE classification and percentage calculated. In Table 1, $29(2.9 \%)$ of the ADRs identified were mainly due to antibiotics which included hypersensitivity reactions, thrombocytopenia, and interstitial nephritis while the other drugs were responsible for electrolyte imbalance, hyperthermia, and nephrotoxicity. Thirty (3\%) serious and $17(1.7 \%)$ significant drug-drug interactions required clinical pharmacist intervention; for which either the offending drug was stopped, dose was reduced, or an alternative drug was prescribed. Remaining 102 (10.4\%) serious and significant drug-drug interactions were closely monitored for any potential manifestation. A total of 135 Y-site drug incompatibilities were detected, of which $8(0.8 \%)$ were visible incompatibilities [Figure 1].

The next most commonly addressed problems were related to drug of choice. Seventy-eight $(8 \%)$ observations in this group to study were due to inappropriate drug or dosage form followed by drug use without clear indication and contraindication of the drug. Drug duplication (drugs of same therapeutic group 


\begin{tabular}{|c|c|c|}
\hline $\begin{array}{l}\text { Primary } \\
\text { domain }\end{array}$ & Detailed classification & $\begin{array}{l}n \text { (percentage } \\
\text { of 986) }\end{array}$ \\
\hline \multirow{3}{*}{$\begin{array}{l}\text { Adverse drug } \\
\text { reactions }\end{array}$} & Antibiotics & $29(2.9)$ \\
\hline & Others & $9(0.9)$ \\
\hline & Total & $38(3.8)$ \\
\hline \multicolumn{3}{|c|}{ Drug interactions Serious drug - drug interactions } \\
\hline & Intervention required & $30(3)$ \\
\hline & Monitoring required & $47(4.8)$ \\
\hline & Significant drug - drug interactions & \\
\hline & Intervention required & $17(\mathrm{I} .7)$ \\
\hline & Monitoring required & $55(5.6)$ \\
\hline & Total & $149(15.1)$ \\
\hline \multirow{3}{*}{$\begin{array}{l}\text { Y-site drug } \\
\text { incompatibility }\end{array}$} & Y-site drug incompatibility (not visible) & $127(12.9)$ \\
\hline & Visible Y-site drug incompatibility & $8(0.8)$ \\
\hline & Total & $135(13.7)$ \\
\hline \multirow{6}{*}{$\begin{array}{l}\text { Drug of choice } \\
\text { problem }\end{array}$} & Inappropriate drug & $13(1.4)$ \\
\hline & Inappropriate dosage form & $65(6.6)$ \\
\hline & Contraindication for drug & $22(2.2)$ \\
\hline & No clear indication of drug use & $45(4.5)$ \\
\hline & No drug prescribed but clear indication & $7(0.7)$ \\
\hline & Total & $152(15.4)$ \\
\hline \multirow[t]{7}{*}{ Drug duplication } & Proton pump inhibitors & $9(0.9)$ \\
\hline & Antihypertensives & $13(1.4)$ \\
\hline & Nebulization drugs & $6(0.6)$ \\
\hline & Statins & $4(0.4)$ \\
\hline & Antibiotics & $5(0.5)$ \\
\hline & Other drugs & $8(0.8)$ \\
\hline & Total & $45(4.6)$ \\
\hline \multirow{5}{*}{$\begin{array}{l}\text { Drug dosing } \\
\text { problem }\end{array}$} & Drug dose too low & $16(1.7)$ \\
\hline & $\begin{array}{l}\text { Drug dose too high or dosage regimen } \\
\text { too frequent }\end{array}$ & $19(1.9)$ \\
\hline & Duration of treatment too short & $3(0.3)$ \\
\hline & Duration of treatment too long & $9(0.9)$ \\
\hline & Total & $47(4.8)$ \\
\hline \multirow{9}{*}{$\begin{array}{l}\text { Medication } \\
\text { errors }\end{array}$} & Improper drug indenting & $8(0.8)$ \\
\hline & Improper dilution technique & $2 I(2.1)$ \\
\hline & Improper administration technique & $95(9.6)$ \\
\hline & Omission of drugs from prescription order & $4 I(4.2)$ \\
\hline & Delayed drug administration & $36(3.7)$ \\
\hline & Incomplete drug labeling & $165(16.7)$ \\
\hline & No or wrong documentation & $7(0.7)$ \\
\hline & Others & $47(4.8)$ \\
\hline & Total & $420(42.6)$ \\
\hline
\end{tabular}

or with same active ingredient) identified by the clinical pharmacist was common with anti-hypertensives and proton pump inhibitors. Drug dosing problems were next on the list with $35(3.6 \%)$ problems due to drug dose too low or too high and $12(1.2 \%)$ problems due to shorter or longer duration of treatment. In Table 1, $42.6 \%$ drug-related problems in our study accounted for medication errors. All medication errors were categorized based on the PCNE classification and were within the benchmarks.

In Table 2, clinical pharmacist interventions for drug-related problems were categorized based on the PCNE classification. A total of 1182 interventions were made by the clinical pharmacist for drug-related problems. Most of the interventions took place at the drug level which was propositions for modification in

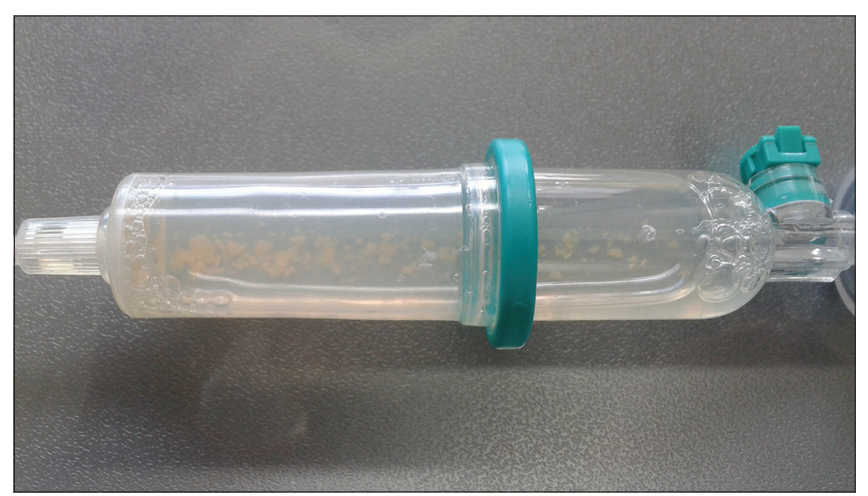

Figure I: Visible Y-site drug incompatibility reported in our Intensive Care Unit between cefepime and vancomycin

therapy. Drug dosing adjustments done by the clinical pharmacist included 140 (11.9\%) renal dose adjustment, $62(5.2 \%)$ hepatic dose adjustment, and $17(1.4 \%)$ pediatric dose adjustment. Glycemic control in the ICU was also under the supervision of the clinical pharmacist which included 104 (8.8\%) insulin dosing modifications. Remaining interventions at drug level included 64 (5.4\%) drug stopped or new drug started, 34 (2.9\%) instruction for drug use changed, and 28 (2.4\%) drug changed and alternatively used. Interventions at the prescriber level comprised prescriber seeking clarification or drug information which was $10.4 \%(n=120)$, and information given to prescriber without seeking clarification was $6.3 \%(n=75)$. A total of 1182 propositions from clinical pharmacist intervention were suggested to the prescriber. About $94.8 \%(n=1121)$ was the acceptance rate for the propositions put forward to the clinician and remaining $5.2 \%(n=61)$ were rejected.

In Table 3, a total of 577 clinical pharmacists answered drug and poison information queries were listed. Majority of the drug information queries regarded drug dosing, indication, and guidelines, which was $34.1 \%(n=197)$. Other queries were regarding ADR, pharmacokinetics, and pharmacodynamics. Poison queries included $47(8.1 \%)$ toxicokinetics, followed by management of poisoning and identification of the poison content.

\section{Discussion}

A critically ill patient is reviewed and treated by physicians from different specialties; hence, polypharmacy is a very common practice in this setting. We presume that medication errors include most commonly transcribing and administration errors which occur at the level of nurses. Indeed, prescribing errors and errors in drug application which occur at the level of the prescriber is often neglected. It is important to have 


\begin{tabular}{|c|c|c|}
\hline $\begin{array}{l}\text { Level of } \\
\text { intervention }\end{array}$ & Detailed classification & $\begin{array}{l}n \text { (percentage } \\
\text { of I I 182) }\end{array}$ \\
\hline \multirow{5}{*}{$\begin{array}{l}\text { At prescriber } \\
\text { level }\end{array}$} & Only information given to prescriber & $75(6.3)$ \\
\hline & $\begin{array}{l}\text { Prescriber asked for clarification or } \\
\text { information }\end{array}$ & $120(10.4)$ \\
\hline & $\begin{array}{l}\text { Intervention proposed, approved by } \\
\text { prescriber }\end{array}$ & $138(|| .5)$ \\
\hline & Intervention proposed but unapproved & $5 \mathrm{I}(4.3)$ \\
\hline & Total & $384(32.5)$ \\
\hline \multirow[t]{6}{*}{ At drug level } & Drug changed & $28(2.4)$ \\
\hline & Dose or dosage form changed & $349(29.5)$ \\
\hline & Instruction for use changed & $34(2.9)$ \\
\hline & Drug stopped & $5 \mid(4.3)$ \\
\hline & New drug started & $13(1.1)$ \\
\hline & Total & $475(40.2)$ \\
\hline Drug dosing & Renal dose adjustment & \\
\hline \multirow[t]{8}{*}{ adjustment done } & Antibiotics & $101(8.6)$ \\
\hline & Other drugs & $39(3.3)$ \\
\hline & Hepatic dose adjustment & \\
\hline & Antibiotics & $46(3.9)$ \\
\hline & Other drugs & $16(1.3)$ \\
\hline & Pediatric dose adjustment & $17(1.4)$ \\
\hline & Insulin dose adjustment & $104(8.8)$ \\
\hline & Total & $323(27.3)$ \\
\hline
\end{tabular}

Table 3: Clinical pharmacist answered drug and poison information queries

\begin{tabular}{llc}
\hline Type of queries & Detailed classification & $\begin{array}{c}\text { n (percentage } \\
\text { of 577) }\end{array}$ \\
\hline Drug information & Dose/indication/guidelines & $197(34.1)$ \\
queries & Pharmacokinetics/dynamics & $74(12.9)$ \\
& Adverse effects & $114(19.8)$ \\
& Other queries & $66(11.4)$ \\
Poison information & Total & $451(78.2)$ \\
queries & Identification of the poison content & $21(3.6)$ \\
& Antidote of poisoning & $13(2.3)$ \\
& Toxic effects of poisoning & $47(8.1)$ \\
& Management of poisoning & $34(5.9)$ \\
& Other queries & $11(1.9)$ \\
& Total & $126(21.8)$ \\
\hline
\end{tabular}

a clinical pharmacist to review the treatment charts to rectify these issues. ${ }^{[10]}$

Numerous published studies show the importance of clinical pharmacist intervention and active participation in multidisciplinary patient care rounds having significantly better patient outcome through quality patient care. ${ }^{[11-13]}$ Interestingly, during the study period, 38 ADRs were reported, and few of them were rare presentations. These results also coincided with a study by Leape who concluded that the participation of clinical pharmacist in medical rounds improved ADR reporting and reduced the rate of preventable ADEs. ${ }^{[6]}$ Many studies from the critical care areas show significant evidence favoring better reporting and prevention of ADEs in the presence of clinical pharmacist. ${ }^{[14,15]}$
The acceptance of the clinical pharmacist's interventions in our study was $94.8 \%$ which was well in accordance with other published data, where the acceptance rate ranges from $85.5 \%$ to $99 \% .{ }^{[16]}$

Critically ill patients are at risk of drug interactions due to the complexity in pharmacotherapy and use of different drug combinations. Drug interactions in these immunocompromised patients can lead to therapeutic failure or ADEs. A study by Reis evaluated the prevalence of potential drug interaction and its clinical significance in critically ill patients. ${ }^{[17,18]}$ The current study managed to contribute substantial information to the physicians regarding the manifested or potential drug interactions in the critical care unit. This included clinical pharmacist interventions due to drug interaction or the need for monitoring the patient.

While most articles focus on drug interactions in critical care, drug incompatibility is also considered likely to harm the patients. As a result of incompatibility, the drug either loses its efficacy or precipitates. About 3.6-18.6\% of incompatible drug combinations have been reported from different studies in the ICU. ${ }^{[19,20]}$ We have also documented 135 Y-site drug incompatibilities which included eight visible incompatibilities.

Streamlining, dose optimization, and de-escalation of antibiotics in the presence of a clinical pharmacist are already proven and recommended to enhance antibiotic stewardship. In our study, clinical pharmacist interventions were effective in rationalizing use of antibiotics, especially in optimizing the dose. ${ }^{[21,22]}$ Plenty of studies have proven reduced incidence of medication errors when pharmacist actively participates in multidisciplinary rounds. ${ }^{[23]}$ In this study, clinical pharmacist not only detected medication errors but also prevented and investigated all types of medication errors.

A national survey by MacLaren et al. from over 260 hospitals, compared clinical and outcome data with the clinical pharmacy services in the ICU. They reported significant impact in terms of mortality, length of stay, and cost benefits in the presence of critical care pharmacist. ${ }^{[24]}$ Parallel studies from MacLaren and Bond using the same data claimed statistically significant clinical and economical outcome associated with thromboembolic or infarct-related events (TIE) in the presence of a clinical pharmacist. ${ }^{[25]}$

Desirable activities of critical care pharmacist also include formulating guidelines for the critically ill patients, active participation in research, and educating 


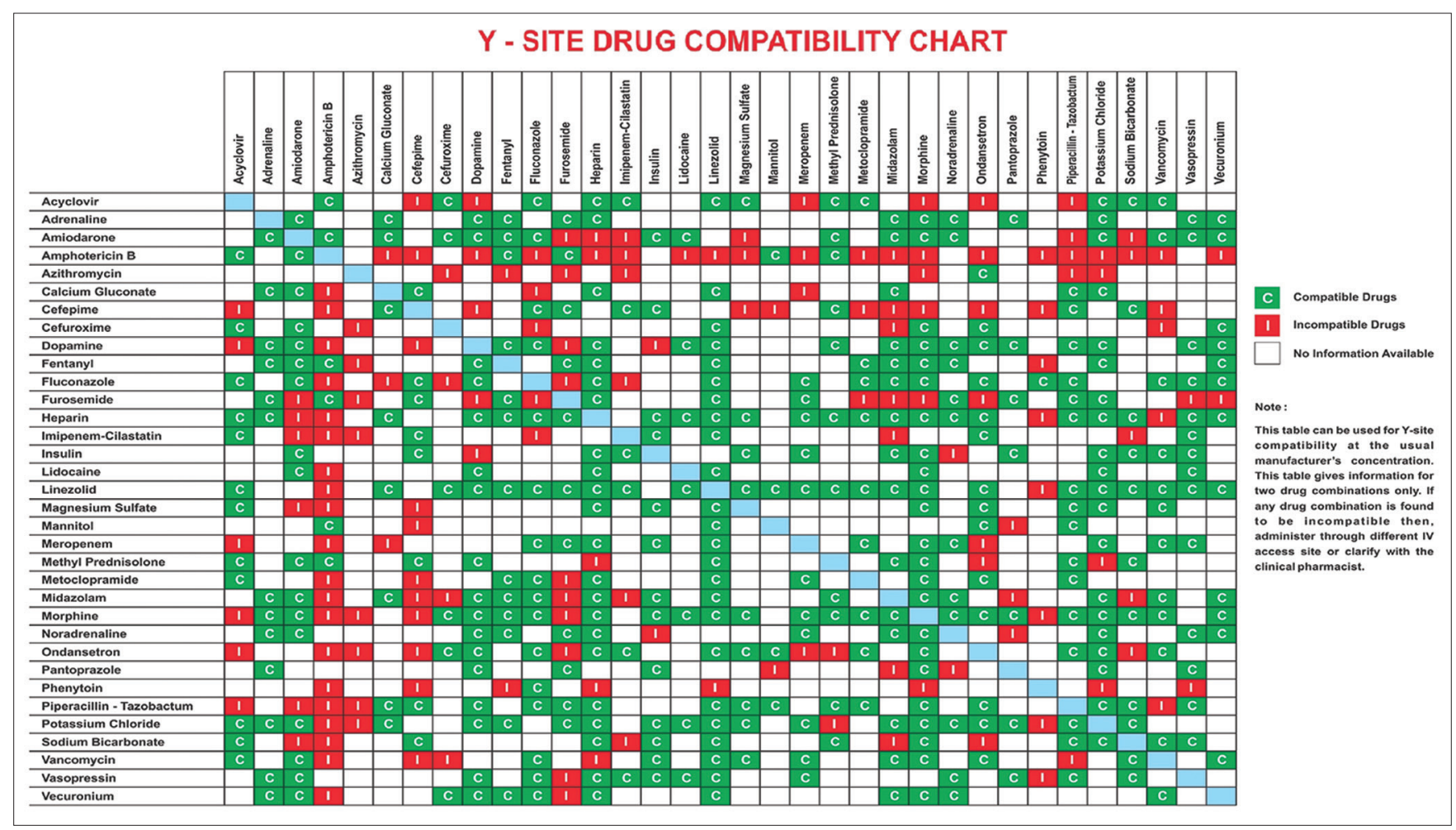

Figure 2: Y-site drug compatibility chart prepared for most commonly used intravenous drugs in Intensive Care Unit

the ICU team. ${ }^{[2]}$ Guidelines which have been developed and implemented by the clinical pharmacist in our ICU includes protocols for pain, sedation, delirium, glycemic control, stress ulcer prophylaxis, deep vein thrombosis prophylaxis, Y-site drug compatibility chart [Figure 2], drug administration, dilution guidelines, and toxicological management protocols. Once the protocols are formulated, all the members of the ICU team are educated on how to use the protocol. Most of these clinical pharmacist enforced protocols are nurse oriented, and hence, it becomes easy for optimizing patient care. The effectiveness of these guidelines is under the supervision of a critical care pharmacist, and it is well studied in Western countries. ${ }^{[25-28]}$

Though our study did not aim to show the economic benefit of clinical pharmacy services, it has definitely reduced drug costs and other treatment costs. Drug cost reduction and treatment cost savings as a result of clinical pharmacist interventions have been assessed and proved in various other studies. ${ }^{[29,30]}$

\section{Conclusions}

Clinical pharmacist as a part of multidisciplinary team in an ICU was associated with a substantially lower rate of adverse drug event caused by medication errors, drug interactions, and drug incompatibilities. Clinical pharmacists are essential to improve patient safety and outcome, reduce costs, and provide quality of care in critically ill patients.

\section{Financial support and sponsorship \\ Nil.}

\section{Conflicts of interest}

There are no conflicts of interest.

\section{References}

1. Kane-Gill SL, Jacobi J, Rothschild JM. Adverse drug events in intensive care units: Risk factors, impact, and the role of team care. Crit Care Med 2010;38 6 Suppl: S83-9.

2. Haupt MT, Bekes CE, Brilli RJ, Carl LC, Gray AW, Jastremski MS, et al. Guidelines on critical care services and personnel: Recommendations based on a system of categorization of three levels of care. Crit Care Med 2003;31:2677-83.

3. Rudis MI, Brandl KM. Position paper on critical care pharmacy services. Society of Critical Care Medicine and American College of Clinical Pharmacy Task Force on Critical Care Pharmacy Services. Crit Care Med 2000;28:3746-50.

4. Boyko WL Jr., Yurkowski PJ, Ivey MF, Armitstead JA, Roberts BL. Pharmacist influence on economic and morbidity outcomes in a tertiary care teaching hospital. Am J Health Syst Pharm 1997;54:1591-5.

5. Smythe MA, Shah PP, Spiteri TL, Lucarotti RL, Begle RL. Pharmaceutical care in medical progressive care patients. Ann Pharmacother 1998;32:294-9.

6. Leape LL, Cullen DJ, Clapp MD, Burdick E, Demonaco HJ, Erickson JI, et al. Pharmacist participation on physician rounds and adverse drug events in the intensive care unit. JAMA 1999;282:267-70.

7. Van Mil JW, Westerlund LO, Hersberger KE, Schaefer MA. Drug-related problem classification systems. Ann Pharmacother 2004;38:859-67.

8. Schaefer M. Discussing basic principles for a coding system of 
drug-related problems: The case of PI-Doc. Pharm World Sci 2002;24:120-7.

9. PCNE.org. Pharmaceutical Care Network Europe, PCNE Classification for Drug Related Problems V6.2; c2010-15. Available from: http://www. pene.org/upload/files/11_PCNE_classification_V6-2.pdf. [Last updated on 2010 Jan 14; Last cited on 2014 Dec 05].

10. Erstad BL, Haas CE, O'Keeffe T, Hokula CA, Parrinello K, Theodorou AA. Interdisciplinary patient care in the intensive care unit: Focus on the pharmacist. Pharmacotherapy 2011;31:128-37.

11. Kim MM, Barnato AE, Angus DC, Fleisher LA, Kahn JM. The effect of multidisciplinary care teams on intensive care unit mortality. Arch Intern Med 2010;170:369-76.

12. Ng TM, Bell AM, Hong C, Hara JM, Touchette DR, Danskey KN, et al. Pharmacist monitoring of QTe interval-prolonging medications in critically ill medical patients: A pilot study. Ann Pharmacother $2008 ; 42: 475-82$.

13. Weant KA, Armitstead JA, Ladha AM, Sasaki-Adams D, Hadar EJ, Ewend MG. Cost effectiveness of a clinical pharmacist on a neurosurgical team. Neurosurgery 2009;65:946-50.

14. Kalina M, Tinkoff G, Gleason W, Veneri P, Fulda G. A multidisciplinary approach to adverse drug events in pediatric trauma patients in an adult trauma center. Pediatr Emerg Care 2009;25:444-6.

15. Jain M, Miller L, Belt D, King D, Berwick DM. Decline in ICU adverse events, nosocomial infections and cost through a quality improvement initiative focusing on teamwork and culture change. Qual Saf Health Care 2006;15:235-9.

16. Klopfer JD, Einarson TR. Acceptance of pharmacists' suggestions by prescribers: A literature review. Hosp Pharm 1990;25:830-2, 834-6.

17. Natasha TG, Carvalho CS, Correia LC, Tenorio DS, Cristina TM, Dias AO, et al. Pharmaceutical intervention assessment in the identification and management of drug interactions in an intensive care unit. J Appl Pharm Sci 2015;5:13-8.

18. Spriet I, Meersseman W, de Hoon J, von Winckelmann S, Wilmer A, Willems L. Mini-series: II. clinical aspects. clinically relevant CYP450-mediated drug interactions in the ICU. Intensive Care Med 2009;35:603-12.

19. Bertsche T, Mayer Y, Stahl R, Hoppe-Tichy T, Encke J, Haefeli WE. Prevention of intravenous drug incompatibilities in an intensive care unit. Am J Health Syst Pharm 2008;65:1834-40.
20. Gikic M, Di Paolo ER, Pannatier A, Cotting J. Evaluation of physicochemical incompatibilities during parenteral drug administration in a paediatric intensive care unit. Pharm World Sci 2000;22:88-91.

21. Klopotowska JE, Kuiper R, van Kan HJ, de Pont AC, Dijkgraaf MG, Lie-A-Huen L, et al. On-ward participation of a hospital pharmacist in a Dutch intensive care unit reduces prescribing errors and related patient harm: An intervention study. Crit Care 2010;14:R174.

22. Scaglione F, Paraboni L. Pharmacokineties/pharmacodynamics of antibacterials in the Intensive Care Unit: Setting appropriate dosing regimens. Int J Antimicrob Agents 2008;32:294-301.

23. Scarsi KK, Fotis MA, Noskin GA. Pharmacist participation in medical rounds reduces medication errors. Am J Health Syst Pharm 2002;59:2089-92.

24. MacLaren R, Bond CA, Martin SJ, Fike D. Clinical and economic outcomes of involving pharmacists in the direct care of critically ill patients with infections. Crit Care Med 2008;36:3184-9.

25. MacLaren R, Bond CA. Effects of pharmacist participation in intensive care units on clinical and economic outcomes of critically ill patients with thromboembolic or infaretion-related events. Pharmacotherapy 2009;29:761-8

26. Marshall J, Finn CA, Theodore AC. Impact of a clinical pharmacist-enforced intensive care unit sedation protocol on duration of mechanical ventilation and hospital stay. Crit Care Med 2008;36:427-33.

27. Kanji S, Singh A, Tierney M, Meggison H, McIntyre L, Hebert PC. Standardization of intravenous insulin therapy improves the efficiency and safety of blood glucose control in critically ill adults. Intensive Care Med 2004:30:804-10.

28. ASHP Therapeutic Guidelines on Stress Ulcer Prophylaxis. ASHP Commission on Therapeuties and approved by the ASHP Board of Directors on November 14, 1998. Am J Health Syst Pharm $1999 ; 56: 347-79$

29. De Rijdt T, Willems L, Simoens S. Economic effects of clinical pharmacy interventions: A literature review. Am J Health Syst Pharm 2008;65:1161-72.

30. Kopp BJ, Mrsan M, Erstad BL, Duby JJ. Cost implications of and potential adverse events prevented by interventions of a critical care pharmacist. Am J Health Syst Pharm 2007;64:2483-7. 